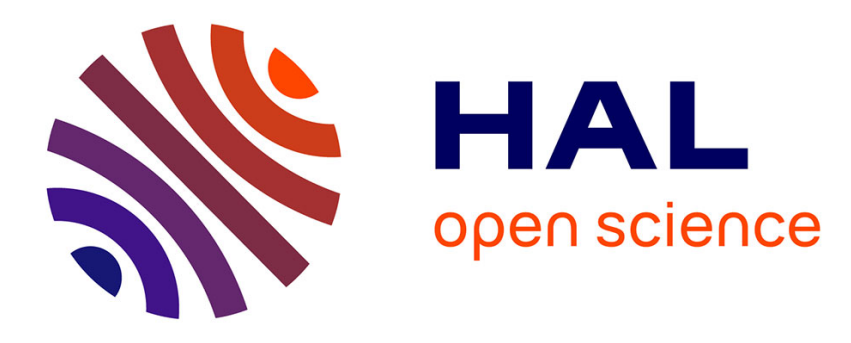

\title{
Null controllability of a linearized Korteweg-de Vries equation by backstepping approach
}

Shengquan Xiang

\section{To cite this version:}

Shengquan Xiang. Null controllability of a linearized Korteweg-de Vries equation by backstepping approach. SIAM Journal on Control and Optimization, 2019, 10.1137/17M1115253 . hal-01468750v2

\section{HAL Id: hal-01468750 \\ https://hal.science/hal-01468750v2}

Submitted on 4 Sep 2018

HAL is a multi-disciplinary open access archive for the deposit and dissemination of scientific research documents, whether they are published or not. The documents may come from teaching and research institutions in France or abroad, or from public or private research centers.
L'archive ouverte pluridisciplinaire HAL, est destinée au dépôt et à la diffusion de documents scientifiques de niveau recherche, publiés ou non, émanant des établissements d'enseignement et de recherche français ou étrangers, des laboratoires publics ou privés. 


\title{
Null controllability of a linearized Korteweg-de Vries equation by backstepping approach
}

\author{
Shengquan Xiang*
}

\begin{abstract}
We prove the null controllability of a linearized Korteweg-de Vries equation with a Dirichlet control on the left boundary. Instead of considering classical methods, i.e. Carleman estimates, moment method etc., we use a backstepping approach which is a method usually used to handle stabilization problems.
\end{abstract}

Keywords. Korteweg-de Vries, backstepping, spectral theory, stabilization, null controllability. AMS Subject Classification. 35Q53, 34H05, 35P10.

\section{Introduction}

We consider the null controllability of the following linearized KdV control system,

$$
\begin{cases}u_{t}(t, x)+u_{x x x}(t, x)+u_{x}(t, x)=0 & \text { in }(0,+\infty) \times(0, L), \\ u(t, L)=u_{x}(t, L)=0 & \text { on }(0,+\infty), \\ u(t, 0)=\kappa(t) & \text { on }(0,+\infty),\end{cases}
$$

where $\kappa(t) \in \mathbb{R}$ is a scalar control.

In [55] Rosier introduced the KdV system with a right boundary Neumann control. One surprisingly finds that controllability depends on the length of the interval, which never happens for the linear finite-dimensional system. More precisely, the system is controllable if and only if

$$
L \notin \mathcal{N}:=\left\{2 \pi \sqrt{\frac{l^{2}+l k+k^{2}}{3}} ; l, k \in \mathbb{N}^{*}\right\} .
$$

This model has been studied for years, in both controllability [7, 8, 10, 15, 17, 55, 57] and stabilization [13, 21, 24, 54, 61].

Concerning the system studied in this paper, we use the left boundary Dirichlet control. For system (1.1), Rosier (see [56]) proved that controllability does not depend on the length of the interval. This system was then further studied in [9,33].

When we study the well-posedness of the control system by using the classical Lions-Magenes method (see [43]), a $H^{1 / 3}$ regularity on the control (with respect to time) is required. Such a problem appears for many boundary control systems, the heat equation and the Burgers equation for example. However, since most control problems are based on evolution models, Sobolev

\footnotetext{
*Sorbonne Université, Université Paris-Diderot SPC, CNRS, INRIA, Laboratoire Jacques-Louis Lions, LJLL, équipe CAGE, F-75005 Paris, France. ETH Zürich, Institute for Theoretical Studies, Forschungsinstitut für Mathematik, 8092 Zürich, Switzerland. E-mail: shengquan.xiang@ens.fr.
} 
type controls are less preferred than piecewise continuous controls (or even $L^{p}$ type conditions), especially for stabilization problems. In [9], Coron and Cerpa proved rapid stabilization of the system (1.1) by using the backstepping method. But since they used some stationary feedback laws, this boundary condition problem is avoided. Recently, by using the (piecewise) backstepping approach, Coron and Nguyen proved the null controllability and semi-global finite time stabilization for a class of heat equations (see [23]). They showed how the use of the maximum principle leads to the well-posedness of the closed-loop system. Their method turns out to be a potential way to solve the local (or even semi-global) finite time stabilization problem for systems which can be rapidly stabilized by means of backstepping methods. At the same time, this method provides a visible way to get null controllability directly instead of using observability inequalities and the duality between controllability and observability.

Initially the backstepping is a method to design stabilizing feedback laws in a recursive manner for systems having a triangular structure. See, for example, [15, Section 12.5]. It was first introduced to deal with finite-dimensional control systems. But it can also be used for control systems modeled by means of partial differential equations (PDEs) as shown first in [18]. For linear partial differential equations, a major innovation is due to Krstic and his collaborators. They observed that, when applied to the classical discretization of these systems, the backstepping leads, at the PDEs level (as the mesh size tends to 0), to the transformation of the initial system into a new target system which can be easily stabilized. This transformation is accomplished by means of a Volterra transform of the second kind. An excellent introduction to this method is presented in [39]. Krstic's innovation has been shown to be very useful for many PDEs control systems as, in particular, heat equations [45, 4, 23], wave equations [60], hyperbolic systems [25, 27, 36, 20] [2, Chapter 7], Korteweg de Vries equations [9, 21], and Kuramoto-Sivashinsky equations [44, 22]. It was observed later on that for some PDEs more general transforms than Volterra transforms of the second kind have to be considered: see $[19,21,22])$. Recently, the backstepping method has been adapted to coupled systems, for example the Boussinesq system of KdV-KdV type [5]. For the case of finite dimensional control system and Krstic's backstepping, see [16].

Krstic's backstepping requires solving a kernel equation. In the case of the heat equation, the kernel equation is a wave equation; however, in this paper the kernel equation turns out to be a third-order equation, which generates new difficulties both for the well-posedness of the closed-loop system and for important estimation issues.

In this paper, we prove that the method developed by Coron and Nguyen can be used to get the null controllability of (1.1).

Theorem 1. For any given $T>0$, the control system (1.1) is null controllable in time $T$ by using some piecewise continuous controls.

Remark 1. Let us recall that the exact controllability of (1.1) fails, which is proved in [56].

Remark 2. We study in detail the well-posedness of the system. The approach and tools introduced for this study do not rely on precise structures. In particular the control is not given by a stationary feedback law (compare to [9]) and no maximum principle is used (compare to [23]). Hence, the well-posedness arguments, as well as a priori estimates, and procedure could easily be adapted to many other partial differential equations.

This paper is organized as follows. Section 2 is a preliminary part including the wellposedness of the systems and the rapid stabilization obtained in [9]. In Section 3, we design the control and provide some estimates which will lead to the null controllability. In Section 
4, we prove the null controllability. We put some further comments in Section 5. It ends with Appendix A (Proposition 1): the proof of the uniqueness of the solution to the kernel equation, which is essential to this paper.

\section{Preliminary}

\subsection{Well-posedness of the control system}

We start with the non-homogeneous linear Cauchy problem

$$
\begin{cases}u_{t}+u_{x x x}+u_{x}=\tilde{h} & \text { in }\left(T_{1}, T_{2}\right) \times(0, L), \\ u_{x}(t, L)=u(t, L)=0 & \text { on }\left(T_{1}, T_{2}\right), \\ u(t, 0)=\kappa(t) & \text { on }\left(T_{1}, T_{2}\right), \\ u(0, x)=u_{0}(x) & \text { on }(0, L),\end{cases}
$$

for

$$
\begin{gathered}
-\infty<T_{1}<T_{2}<+\infty, \\
u_{0} \in L^{2}(0, L), \\
\tilde{h} \in L^{1}\left(T_{1}, T_{2} ; L^{2}(0, L)\right), \\
\kappa \in L^{2}\left(T_{1}, T_{2}\right) .
\end{gathered}
$$

Definition 1. A solution to the Cauchy problem (2.1)-(2.5) is a function $u \in C^{0}\left(\left[T_{1}, T_{2}\right] ; L^{2}(0, L)\right)$ such that, for every $\tau \in\left[T_{1}, T_{2}\right]$ and for every $\phi \in C^{3}\left(\left[T_{1}, \tau\right] \times[0, L]\right)$ satisfying

$$
\phi(t, 0)=\phi(t, L)=\phi_{x}(t, 0)=0, \forall t \in\left[T_{1}, \tau\right],
$$

one has

$$
\begin{gathered}
-\int_{T_{1}}^{\tau} \int_{0}^{L}\left(\phi_{t}+\phi_{x}+\phi_{x x x}\right) u d x d t-\int_{T_{1}}^{\tau} \kappa(t) \phi_{x x}(t, 0) d t-\int_{T_{1}}^{\tau} \int_{0}^{L} \phi \tilde{h} d x d t \\
+\int_{0}^{L} u(\tau, x) \phi(\tau, x) d x-\int_{0}^{L} u_{0} \phi\left(T_{1}, x\right) d x=0 .
\end{gathered}
$$

The uniqueness of the solution to the Cauchy problem (2.1)-(2.5) is straightforward, one can get details from the book by Coron [15]. For the existence of the solution, in [3], Bona, Sun, and Zhang proved the following result.

Lemma 1. If $h \in H^{1 / 3}\left(T_{1}, T_{2}\right)$, then the Cauchy problem (2.1) has one and only one solution. This solution is in $C^{0}\left(\left[T_{1}, T_{2}\right] ; L^{2}(0, L)\right) \cap L^{2}\left(T_{1}, T_{2} ; H^{1}(0, L)\right)$. There exists a constant $c_{1}>0$ depending on $\left(T_{2}-T_{1}\right)$ such that

$$
\begin{aligned}
\|u\|_{C^{0}\left(\left[T_{1}, T_{2}\right] ; L^{2}(0, L)\right)} & +\|u\|_{L^{2}\left(T_{1}, T_{2} ; H^{1}(0, L)\right)}+\sup _{x \in[0, L]}\left\|u_{x}(\cdot, x)\right\|_{L^{2}\left(T_{1}, T_{2}\right)} \\
& \leqslant c_{1}\left(\left\|u_{0}\right\|_{L^{2}(0, L)}+\|\kappa\|_{H^{1 / 3}\left(T_{1}, T_{2}\right)}+\|\tilde{h}\|_{L^{1}\left(T_{1}, T_{2} ; L^{2}(0, L)\right)}\right) .
\end{aligned}
$$




\subsection{Rapid stabilization of (1.1)}

We recall some results given in [9]. Given a positive parameter $\lambda>1$, we consider the following equations in the triangle $\mathcal{T}:=\{(x, y): x \in[0, L], y \in[x, L]\}$,

$$
\begin{cases}k_{x x x}+k_{y y y}+k_{x}+k_{y}+\lambda k=0 & \text { in } \mathcal{T}, \\ k(x, L)=0 & \text { on }[0, L], \\ k(x, x)=0 & \text { on }[0, L], \\ k_{x}(x, x)=\frac{\lambda}{3}(L-x) & \text { on }[0, L],\end{cases}
$$

and

$$
\begin{cases}l_{x x x}+l_{y y y}+l_{x}+l_{y}-\lambda l=0 & \text { in } \mathcal{T}, \\ l(x, L)=0 & \text { on }[0, L], \\ l(x, x)=0 & \text { on }[0, L], \\ l_{x}(x, x)=\frac{\lambda}{3}(L-x) & \text { on }[0, L] .\end{cases}
$$

In [9], it is noted that both (2.9) and (2.10) have solutions in $C^{3}(\mathcal{T})$. These solutions are further studied in Section 3, where we provide some estimates on $\|k\|_{C^{0}(\mathcal{T})}$ with respect to $\lambda$. Actually, the solutions of equation (2.9) and of (2.10) satisfy the following conditions

$$
\begin{aligned}
& k_{x y}(x, x)=-\frac{\lambda}{3}, \\
& l_{x y}(x, x)=-\frac{\lambda}{3},
\end{aligned}
$$

respectively. The Properties (2.11) and (2.12) can be checked as follows: we perform the change of variables,

$$
t=y-x, \quad s=x+y,
$$

and define

$$
G(s, t):=k(x, y) .
$$

Then equation (2.9) of $k$ becomes the following equation of $G$,

$$
\begin{cases}6 G_{t t s}+2 G_{s s s}+2 G_{s}+\lambda G=0 & \text { in } \mathcal{T}_{0}, \\ G(s, 2 L-s)=0 & \text { on }[L, 2 L], \\ G(s, 0)=0 & \text { on }[0,2 L], \\ G_{t}(s, 0)=\frac{\lambda}{6}(s-2 L) & \text { on }[0,2 L],\end{cases}
$$

where $\mathcal{T}_{0}:=\{(s, t) ; t \in[0, L], s \in[t, 2 L-t]\}$. From (2.15), one easily gets

$$
G_{t t s}(s, 0)=0 \text { in }[0,2 L] \text {. }
$$

Hence, $G_{t t}(s, 0)=G_{t t}(2 L, 0)$. In order to calculate $G_{t t}(2 L, 0)$, one observes from (2.15) that

$$
G_{t t}(2 L, 0)=2 G_{t s}(2 L, 0)=\frac{\lambda}{3} .
$$

Direct calculations show that

$$
k_{x y}(x, x)=-G_{t t}(s, 0)=-\frac{\lambda}{3},
$$


which concludes (2.11). The proof of (2.12) is similar.

Now, let us define a continuous transformation $\Pi_{\lambda}: L^{2}(0, L) \rightarrow L^{2}(0, L)$ by

$$
\omega(x)=\Pi_{\lambda}(u(x)):=u(x)-\int_{x}^{L} k(x, y) u(y) d y .
$$

Moreover, its inverse is given by (let us denote by $\Pi_{\lambda}^{-1}$ )

$$
u(x)=\Pi_{\lambda}^{-1}(\omega(x)):=\omega(x)+\int_{x}^{L} l(x, y) \omega(y) d y .
$$

That is because $k(x, y)$ and $l(x, y)$ are related by the formula

$$
l(x, y)-k(x, y)=\int_{x}^{y} k(x, \eta) l(\eta, y) d \eta
$$

Actually, one can define

$$
\tilde{l}(x, y):=k(x, y)+\int_{x}^{y} k(x, \eta) l(\eta, y) d \eta
$$

Hence one only needs to prove $l=\tilde{l}$ to get (2.21). Direct calculations show that $\tilde{l}$ satisfies

$$
\begin{cases}\tilde{l}_{x x x}+\tilde{l}_{y y y}+\tilde{l}_{x}+\tilde{l}_{y}-\lambda l=0 & \text { in } \mathcal{T}, \\ \tilde{l}(x, L)=0 & \text { on }[0, L], \\ \tilde{l}(x, x)=0 & \text { on }[0, L], \\ \tilde{l}_{x}(x, x)=\frac{\lambda}{3}(L-x) & \text { on }[0, L], \\ \tilde{l}_{x y}(x, x)=-\frac{\lambda}{3} & \text { on }[0, L] .\end{cases}
$$

Let us define $l_{0}:=l-\tilde{l}$. From (2.23), (2.10), and (2.12), one knows that

$$
\begin{cases}\left(l_{0}\right)_{x x x}+\left(l_{0}\right)_{y y y}+\left(l_{0}\right)_{x}+\left(l_{0}\right)_{y}=0 & \text { in } \mathcal{T}, \\ \left(l_{0}\right)(x, L)=0 & \text { on }[0, L], \\ \left(l_{0}\right)(x, x)=0 & \text { on }[0, L], \\ \left(l_{0}\right)_{x}(x, x)=0 & \text { on }[0, L], \\ \left(l_{0}\right)_{x y}(x, x)=0 & \text { on }[0, L] .\end{cases}
$$

Regarding to the Cauchy problem (2.24), we have the following proposition (hence $l=\tilde{l}$ ), whose proof is given in Appendix A.

Proposition 1. The equation (2.24) has a unique solution in $C^{3}(\mathcal{T})$. More precisely, this solution is $l_{0}=0$.

Remark 3. This proposition is important to this paper. In the following section we construct precisely a solution to equation (2.9)(and of (2.10) respectively), the proof of Theorem 1 relies on some estimates of this solution. Proposition 1 ensures the solution that we construct satisfies (2.21) (hence (2.20)). 
We find that by using the transformation $\Pi_{\lambda}$, the solution of (1.1) with control

$$
\kappa(t)=\int_{0}^{L} k(0, y) u(t, y) d y
$$

is mapped to a solution of the system

$$
\begin{cases}\omega_{t}+\omega_{x x x}+\omega_{x}+\lambda \omega=0 & \text { in }(0,+\infty) \times(0, L), \\ \omega(t, L)=\omega_{x}(t, L)=0 & \text { on }(0,+\infty), \\ \omega(t, 0)=0 & \text { on }(0,+\infty) .\end{cases}
$$

For system (2.26), one can easily obtain exponential decay of the solution

$$
\|\omega(t, \cdot)\|_{L^{2}(0, L)} \leqslant e^{-\lambda t}\|\omega(0, \cdot)\|_{L^{2}(0, L)} .
$$

Hence the solution of (1.1) with feedback law (2.25) satisfies

$$
\begin{aligned}
\|u(t, \cdot)\|_{L^{2}(0, L)} & \leqslant\left\|\Pi_{\lambda}^{-1}\right\|_{L^{2}(0, L) \rightarrow L^{2}(0, L)}\|\omega(t, \cdot)\|_{L^{2}(0, L)} \\
& \leqslant e^{-\lambda t}\left\|\Pi_{\lambda}^{-1}\right\|_{L^{2}(0, L) \rightarrow L^{2}(0, L)}\|\omega(0, \cdot)\|_{L^{2}(0, L)} \\
& \leqslant e^{-\lambda t}\left\|\Pi_{\lambda}^{-1}\right\|_{L^{2}(0, L) \rightarrow L^{2}(0, L)}\left\|\Pi_{\lambda}\right\|_{L^{2}(0, L) \rightarrow L^{2}(0, L)}\|u(0, \cdot)\|_{L^{2}(0, L)} .
\end{aligned}
$$

From now on, we simply denote $\left\|\Pi_{\lambda}^{-1}\right\|_{L^{2}(0, L) \rightarrow L^{2}(0, L)}$ by $\left\|\Pi_{\lambda}^{-1}\right\|$ to simplify the notations.

\subsection{Well-posedness of system (2.26)}

For a positive parameter $\lambda>0$, we consider the following linear operator $\mathcal{A}_{\lambda}: \mathcal{D}\left(\mathcal{A}_{\lambda}\right) \subset$ $L^{2}(0, L) \rightarrow L^{2}(0, L)$ with

$$
\begin{gathered}
\mathcal{D}\left(\mathcal{A}_{\lambda}\right):=\left\{f \in H^{3}(0, L) ; f(0)=f(L)=f_{x}(L)=0\right\}, \\
\mathcal{A}_{\lambda}:=-f_{x}-f_{x x x}-\lambda f, \forall f \in \mathcal{D}\left(\mathcal{A}_{\lambda}\right) .
\end{gathered}
$$

Similarly, for the case where $\lambda=0$ (see [15, page 38-43]), the following properties also hold.

$$
\begin{gathered}
\mathcal{D}\left(\mathcal{A}_{\lambda}\right) \text { is dense in } L^{2}(0, L), \\
\mathcal{A}_{\lambda} \text { is closed, } \\
\mathcal{A}_{\lambda} \text { and } A^{*} \text { are dissipative. }
\end{gathered}
$$

Hence, $\mathcal{A}_{\lambda}$ generates a strongly continuous semigroup of linear operator $\left\{S_{\lambda}(t)\right\}_{t \geqslant 0}$ on $L^{2}(0, L)$. Furthermore, for every initial data $\omega_{0} \in \mathcal{D}\left(\mathcal{A}_{\lambda}\right)$, system (2.26) has one and only one solution $\omega(t, x) \in C^{0}\left([0,+\infty) ; L^{2}(0, L)\right)$. This solution also satisfies

$$
\begin{gathered}
\omega \in C^{1}\left([0,+\infty) ; L^{2}(0, L)\right) \cap C^{0}\left([0,+\infty) ; \mathcal{D}\left(\mathcal{A}_{\lambda}\right)\right), \\
\left\|\omega_{x}(\cdot, 0)\right\|_{L^{2}(0, T)} \leqslant\left\|\omega_{0}\right\|_{L^{2}(0, L)}, \forall T>0, \\
\|\omega\|_{L^{2}\left(0, T ; H^{1}(0, L)\right)} \leqslant C_{T}\left\|\omega_{0}\right\|_{L^{2}(0, L)}, \text { where } C_{T} \text { only depends on } \mathrm{T}>0 .
\end{gathered}
$$

By standard approximation arguments, it follows that when $\omega_{0} \in L^{2}(0, L)$ equation $(2.26)$ has a unique solution $\omega(t, x) \in C^{0}\left([0,+\infty) ; L^{2}(0, L)\right)$. This solution also satisfies $(2.35)$ and (2.36). For more details on the results and proofs of this subsection, one can refer to Coron's book [15, page 38-43 and page 374-377]. Although the book only describes the case when $\lambda=0$, the general case $\lambda>0$ follows by considering $e^{\lambda t} \omega$.

Remark 4. Inequality (2.35) is a hidden inequality. It was first found by Rosier in [55]. Inequality (2.36) is the Kato smoothing effect. 


\section{Control design}

Inspired by the work of Coron and Nguyen in [23], we construct a piecewise control such that on each piece, the solution of (1.1) can be transformed to a solution of (2.26). More precisely, we select

$\left\{\lambda_{n}\right\}_{n \in \mathbb{N}}$, increasing positive numbers that tends to infinity,

$\left\{t_{n}\right\}_{n \in \mathbb{N}}$, increasing numbers with $t_{0}=0$ that tends to $T$ as $n$ tends to infinity.

First we define

$$
u(0):=u_{0} \text { and } u(T):=0 .
$$

Then, for $t_{n}<t \leqslant t_{n+1}$, we successively define

$$
\begin{gathered}
u(t):=\Pi_{\lambda_{n}}^{-1} S_{\lambda_{n}}\left(t-t_{n}\right) \Pi_{\lambda_{n}} u\left(t_{n}\right), \\
\kappa(t):=\int_{0}^{L} k_{\lambda_{n}}(0, y) u(t, y) d y,
\end{gathered}
$$

where $S_{\lambda_{n}}$ is the semigroup given in Section 2.3.

One has the following lemma, whose proof is given at the end of this section.

Lemma 2. As defined in (3.3)-(3.4), $\left.u(t)\right|_{t_{n} \leqslant t \leqslant t_{n+1}}$ is a solution of (2.1) with $T_{1}=t_{n}, T_{2}=$ $t_{n+1}, \tilde{h}=0$, and $\kappa(t)$ given by $(3.5)$.

Let us define

$$
s_{0}:=0 \text { and } s_{n}:=\sum_{k=0}^{n-1} \lambda_{k}\left(t_{k+1}-t_{k}\right) \text { for } n \geqslant 1,
$$

thanks to (3.4) and (2.28), we get

$$
\begin{gathered}
\|u(t, \cdot)\|_{L^{2}(0, L)} \leqslant e^{-s_{n}}\left\|u_{0}\right\|_{L^{2}(0, L)} \prod_{k=0}^{n}\left(\left\|\Pi_{\lambda_{k}}^{-1}\right\|\left\|\Pi_{\lambda_{k}}\right\|\right), \\
|\kappa(t)| \leqslant e^{-s_{n}}\left\|u_{0}\right\|_{L^{2}(0, L)}\left\|k_{\lambda_{n}}(0, \cdot)\right\|_{L^{2}(0, L)} \prod_{k=0}^{n}\left(\left\|\Pi_{\lambda_{k}}^{-1}\right\|\left\|\Pi_{\lambda_{k}}\right\|\right)
\end{gathered}
$$

for $t \in\left[t_{n}, t_{n+1}\right]$.

Hence, if we have a good estimation on $k_{\lambda}$, it will be possible to get $u(t) \rightarrow 0$ when $t$ tends to $T$. Actually, we have the following estimates.

Lemma 3. Let $\lambda>2$, then (2.9) has a unique solution $k_{\lambda} \in C^{3}(\mathcal{T})$ (respectively $(2.10)$ has a unique solution $l_{\lambda} \in C^{3}(\mathcal{T})$ ). Those solutions also satisfy

$$
\left\|k_{\lambda}\right\|_{C^{0}(\mathcal{T})} \leqslant e^{(1+L)^{2} \sqrt{\lambda}} \text { and }\left\|l_{\lambda}\right\|_{C^{0}(\mathcal{T})} \leqslant e^{(1+L)^{2} \sqrt{\lambda}}
$$

Proof of Lemma 3. The existence of solution to (2.9) is given in [9]. The uniqueness of the solution is proved in Appendix A. Here we focus on the $C^{0}$ norm estimate (3.9).

Take the following change of variable,

$$
t=y-x, \quad s=x+y
$$


and define

$$
G(s, t):=k(x, y) .
$$

Then we transform (2.9) into an integral equation of $G(s, t)$ (see formula (21) in [9]),

$$
\begin{aligned}
& G(s, t)=-\frac{\lambda t}{6}(2 L-t-s) \\
& +\frac{1}{6} \int_{s}^{2 L-t} \int_{0}^{t} \int_{0}^{\tau}\left(2 G_{s s s}+2 G_{s}+\lambda G\right)(\eta, \xi) d \xi d \tau d \eta,
\end{aligned}
$$

in $\mathcal{T}_{0}:=\{(s, t) ; t \in[0, L], s \in[t, 2 L-t]\}$.

We use a successive approximation to give a solution of the equation (3.12). Thanks to Proposition 1, this solution is the unique solution of (3.12). Let us take

$$
G^{1}(s, t):=-\frac{\lambda t}{6}(2 L-t-s)
$$

and define

$$
G^{n+1}(s, t)=\frac{1}{6} \int_{s}^{2 L-t} \int_{0}^{t} \int_{0}^{\tau}\left(2 G_{s s s}^{n}+2 G_{s}^{n}+\lambda G^{n}\right)(\eta, \xi) d \xi d \tau d \eta .
$$

For instance,

$$
G^{2}(s, t)=\frac{1}{108}\left\{t^{3}\left(\lambda-\lambda^{2} L+\frac{\lambda^{2} t}{4}\right)(2 L-t-s)+\frac{t^{3} \lambda^{2}}{4}\left[(2 L-t)^{2}-s^{2}\right]\right\} .
$$

But unfortunately, we can not perform such explicit calculation by hand each time. We try to estimate $G^{n}(s, t)$ from another way. Notice that if $f(s, t):=g(s) h(t)$, then

$$
\begin{aligned}
& \int_{s}^{2 L-t} \int_{0}^{t} \int_{0}^{\tau} \frac{\partial^{m}}{\partial s^{m}} f(\eta, \xi) d \xi d \tau d \eta \\
= & \int_{s}^{2 L-t} \int_{0}^{t} \int_{0}^{\tau} \frac{\partial^{m}}{\partial s^{m}} g(\eta) h(\xi) d \xi d \tau d \eta \\
= & \int_{s}^{2 L-t} \frac{\partial^{m}}{\partial s^{m}} g(\eta) d \eta \int_{0}^{t} \int_{0}^{\tau} h(\xi) d \xi d \tau .
\end{aligned}
$$

Let $\mathbb{P}$ be the space of polynomials of one variable on $\mathbb{R}$. We define operator $\mathbf{T}$ by

$$
\begin{aligned}
\mathbf{T}: \mathbb{P} \otimes \mathbb{P} & \rightarrow \mathbb{P} \otimes \mathbb{P} \\
g(s) h(t) & \mapsto \frac{1}{6} \int_{s}^{2 L-t} \int_{0}^{t} \int_{0}^{\tau}\left(2 \frac{\partial^{3}}{\partial s^{3}}+2 \frac{\partial}{\partial s}+\lambda I d\right)(g \cdot h)(\eta, \xi) d \xi d \tau d \eta .
\end{aligned}
$$

Equality (3.16) shows that (3.17) is well defined. In fact

$$
\mathbf{T}(g(s) h(t))=g_{\mathbf{T}}(s, t) h_{\mathbf{T}}(t),
$$

where $g_{\mathbf{T}}(s, t)$ and $h_{\mathbf{T}}(t)$ are given by

$$
\begin{gathered}
g_{\mathbf{T}}(s, t):=\frac{1}{6} \int_{s}^{2 L-t}\left(2 \frac{\partial^{3}}{\partial s^{3}}+2 \frac{\partial}{\partial s}+\lambda I d\right)(g)(\eta) d \eta \\
h_{\mathbf{T}}(t):=\int_{0}^{t} \int_{0}^{\tau} h(\xi) d \xi d \tau .
\end{gathered}
$$


Observe that, if

$$
|h(t)| \leqslant t^{n}, \forall 0 \leqslant t \leqslant L,
$$

then

$$
\left|h_{\mathbf{T}}(t)\right|=\left|\int_{0}^{t} \int_{0}^{\tau} h(\xi) d \xi d \tau\right| \leqslant \frac{1}{(n+1)(n+2)} t^{n+2}, \quad \text { for } t \in[0, L] .
$$

As for $g_{\mathbf{T}}(s, t)$, notice that if $g(s)=s^{m}$ with $m \geqslant 3$, then

$$
\begin{aligned}
g_{\mathbf{T}}(s, t)= & \frac{1}{6}\left(2 m(m-1)(2 L-t)^{m-2}-2 m(m-1) s^{m-2}\right. \\
& \left.+2(2 L-t)^{m}-2 s^{m}+\frac{\lambda(2 L-t)^{m+1}}{m+1}-\frac{\lambda s^{m+1}}{m+1}\right) .
\end{aligned}
$$

This inspires us to separate $\mathbf{T}$ into the following 6 linear operators $\left\{\mathbf{T}_{i}\right\}_{1 \leqslant i \leqslant 6}$ from $\mathbb{P} \otimes \mathbb{P}$ to $\mathbb{P} \otimes \mathbb{P}$.

$$
\begin{gathered}
\mathbf{T}_{1}: s^{m} h(t) \mapsto \begin{cases}\frac{1}{6}\left(2 m(m-1)(2 L-t)^{m-2}\right) h_{\mathbf{T}}(t), & \text { when } m \geqslant 3, \\
0, & \text { when } 0 \leqslant m \leqslant 2,\end{cases} \\
\mathbf{T}_{2}: s^{m} h(t) \mapsto \begin{cases}-\frac{1}{6}\left(2 m(m-1) s^{m-2}\right) h_{\mathbf{T}}(t), & \text { when } m \geqslant 3, \\
0, & \text { when } 0 \leqslant m \leqslant 2,\end{cases} \\
\mathbf{T}_{3}: s^{m} h(t) \mapsto \begin{cases}\frac{1}{6}\left(2(2 L-t)^{m}\right) h_{\mathbf{T}}(t), & \text { when } m \geqslant 1, \\
0, & \text { when } m=0,\end{cases} \\
\mathbf{T}_{4}: s^{m} h(t) \mapsto \begin{cases}-\frac{1}{6}\left(2 s^{m}\right) h_{\mathbf{T}}(t), & \text { when } m \geqslant 1, \\
0, & \text { when } m=0,\end{cases} \\
\mathbf{T}_{5}: s^{m} h(t) \mapsto \frac{\lambda}{6} \frac{(2 L-t)^{m+1}}{m+1} h_{\mathbf{T}}(t), \\
\text { when } m \geqslant 0,
\end{gathered}
$$

Since $\mathbf{T}_{i}$ is linear, we easily find that

$$
\mathbf{T}_{i}(0)=0
$$

From (3.17)-(3.29), we know that

$$
\mathbf{T}=\sum_{i=1}^{6} \mathbf{T}_{i}, \text { on } \mathbb{P} \otimes \mathbb{P} .
$$

Hence,

$$
\begin{aligned}
G^{n+1}(s, t) & =\mathbf{T} G^{n}(s, t) \\
& =\left(\sum_{i=1}^{6} \mathbf{T}_{i}\right) G^{n}(s, t) \\
& =\left(\sum_{i=1}^{6} \mathbf{T}_{i}\right)^{n} G^{1}(s, t) .
\end{aligned}
$$


By (3.13), we get

$$
G^{1}(s, t)=-\frac{\lambda}{6} t(2 L-t)+\frac{\lambda}{6}(s t)=I(s, t)+J(s, t),
$$

where

$$
I(s, t):=-\frac{\lambda}{6} t(2 L-t) \text { and } J(s, t):=\frac{\lambda}{6}(s t)
$$

Let us define

$$
\mathbb{A}_{n}:=\left\{\left(x_{1}, x_{2}, \ldots, x_{n}\right) ; x_{i} \in\{1,2,3,4,5,6\}, \forall 1 \leqslant i \leqslant n\right\}, \forall n \geqslant 1 .
$$

For any $n \in \mathbb{N}^{*}$, for any $a=(a(1), a(2), \ldots, a(n)) \in \mathbb{A}_{n}$, we define the operator

$$
\mathbf{T}_{a}:=\mathbf{T}_{a(n)} \mathbf{T}_{a(n-1)} \ldots \mathbf{T}_{a(1)}
$$

We define additionally $\mathbb{A}:=\left\{a_{0}\right\}$ and $\mathbf{T}_{a_{0}}:=I d$ (identity operator on $\mathbb{P}$ ).

Hence for any $n \in \mathbb{N}$, we have

$$
\begin{aligned}
G^{n+1}(s, t) & =\mathbf{T}^{n}(I+J) \\
& =\sum_{a \in \mathbb{A}_{n}}\left(\mathbf{T}_{a} I\right)+\sum_{a \in \mathbb{A}_{n}}\left(\mathbf{T}_{a} J\right) .
\end{aligned}
$$

Now we use mathematical induction to conclude the following lemma.

Lemma 4. For every $\lambda>2$, for every $n \in \mathbb{N}$ and for every $a \in \mathbb{A}_{n}, \mathbf{T}_{a} I$ and $\mathbf{T}_{a} J$ are of the form $s^{l} h(t)$. They also satisfy

$$
|h(t)| \leqslant\left(\frac{\lambda}{6}\right)^{n+1} \frac{t^{2 n+1}}{(2 n+1) !}(2 L+1)^{n+1-l} \frac{1}{l !}, \quad t \in[0, L] .
$$

Proof of Lemma 4. When $n=0$, one can check that Lemma 4 holds. Let us suppose that Lemma 4 holds when $n=k \geqslant 0$. Then we can check in the rest of the proof that Lemma 4 holds when $n=k+1$.

For any $n \geqslant 1$, and for any $a:=(a(1), a(2), \ldots, a(n+1)) \in \mathbb{A}_{n+1}$, let us define

$$
\varrho(a):=(a(1), a(2), \ldots, a(n)) .
$$

For any $a \in \mathbb{A}_{1}$, let us define

$$
\varrho(a):=a_{0} .
$$

Hence for any $a:=(a(1), a(2), \ldots, a(k+1)) \in \mathbb{A}_{k+1}$, we have

$$
\mathbf{T}_{a}=\mathbf{T}_{a(k+1)} \mathbf{T}_{\varrho(a)}
$$

From the assumption, we know that

$$
\mathbf{T}_{\varrho(a)} I=s^{l} h(t) .
$$

If $\mathbf{T}_{\varrho(a)} I=0$, then we conclude the proof.

If $\mathbf{T}_{\varrho(a)} I=s^{l} h(t)$, then we know from (3.37) that

$$
|h(t)| \leqslant\left(\frac{\lambda}{6}\right)^{k+1} \frac{t^{2 k+1}}{(2 k+1) !}(2 L+1)^{1+k-l} \frac{1}{l !}, \quad t \in[0, L] .
$$


Let us first consider $\mathbf{T}_{1}$. We know that $\mathbf{T}_{1}\left(s^{l} h(t)\right)=0$ if $l \leqslant 2$. Therefore, it suffices to prove the case when $l \geqslant 3$.

From (3.20)-(3.22), (3.24), and (3.42), we know that

$$
\begin{aligned}
\left|\mathbf{T}_{1}\left(s^{l} h(t)\right)\right| & =\left|\frac{2}{6}\left(l(l-1)(2 L-t)^{l-2}\right) h_{\mathbf{T}}(t)\right| \\
& \leqslant \frac{2}{6}\left(l(l-1)(2 L-t)^{l-2}\right)\left(\frac{\lambda}{6}\right)^{1+k} \frac{t^{2 k+3}}{(2 k+3) !}(2 L+1)^{1+k-l} \frac{1}{l !} \\
& \leqslant\left(\frac{\lambda}{6}\right)^{k+2} \frac{t^{2 k+3}}{(2 k+3) !}(2 L+1)^{k-1} \frac{1}{(l-2) !} .
\end{aligned}
$$

Notice that $\mathbf{T}_{1}\left(s^{l} h(t)\right)$ can be written as $s^{0} g(t)$. Thus, it can be seen from (3.43) that (3.37) is satisfied.

By using the same procedure, we can check that

$$
\begin{aligned}
\left|\mathbf{T}_{2}\left(s^{l} h(t)\right)\right| & \leqslant\left(\frac{\lambda}{6}\right)^{k+2} \frac{t^{2 k+3}}{(2 k+3) !}(2 L+1)^{1+k-l} \frac{s^{l-2}}{(l-2) !}, \\
\left|\mathbf{T}_{3}\left(s^{l} h(t)\right)\right| & \leqslant\left(\frac{\lambda}{6}\right)^{k+2} \frac{t^{2 k+3}}{(2 k+3) !}(2 L+1)^{1+k} \frac{1}{l !}, \\
\left|\mathbf{T}_{4}\left(s^{l} h(t)\right)\right| & \leqslant\left(\frac{\lambda}{6}\right)^{k+2} \frac{t^{2 k+3}}{(2 k+3) !}(2 L+1)^{1+k-l} \frac{s^{l}}{l !}, \\
\left|\mathbf{T}_{5}\left(s^{l} h(t)\right)\right| & \leqslant\left(\frac{\lambda}{6}\right)^{k+2} \frac{t^{2 k+3}}{(2 k+3) !}(2 L+1)^{k+2} \frac{1}{(l+1) !}, \\
\left|\mathbf{T}_{6}\left(s^{l} h(t)\right)\right| & \leqslant\left(\frac{\lambda}{6}\right)^{k+2} \frac{t^{2 k+3}}{(2 k+3) !}(2 L+1)^{1+k-l} \frac{s^{l+1}}{(l+1) !}
\end{aligned}
$$

Hence, we complete the proof.

By the same idea of partition and Lemma 4, we can further obtain the following estimate.

Lemma 5. For every $\lambda>2$, for every $n \in \mathbb{N}$, and for every $a \in \mathbb{A}_{n}, \mathbf{T}_{a} I$ and $\mathbf{T}_{a} J$ are of the form $s^{l} h(t)$. They also satisfy

$$
\left|\partial_{t}(h(t))\right| \leqslant 2\left(\frac{\lambda}{6}\right)^{n+1} \frac{t^{2 n}}{(2 n) !}(2 L+1)^{n+3-l} \frac{1}{l !}, \quad t \in[0, L] .
$$

Remark 5. One can get similar estimates for $C^{2}$-norm or even $C^{n}$-norm. However, since in this paper we do not need to use such estimates, this part is omitted. Actually, $C^{2}$-norm estimates can be obtained directly by Lemma 4 (as the way of getting Lemma 5), but the $C^{n}$ norm (with $n \geq 3$ ) is more complicated. Furthermore, it can be seen from [39] that for the heat equation the kernel is analytic in the triangle. It is of interest to know if the kernel we obtained in this article is also analytic.

We come back to the estimate (3.36). From Lemma 4, we know that, for every $n \in \mathbb{N}$, for every $a \in \mathbb{A}_{n}$, for every $m \in \mathbb{N}$, and for $t \in[0, L]$ we have

$$
\begin{aligned}
& \left|\frac{\partial^{m}}{\partial s^{m}}\left(\mathbf{T}_{a} I\right)(s, t)\right| \leqslant\left(\frac{\lambda}{6}\right)^{1+n} \frac{t^{2 n+1}}{(2 n+1) !}(2 L+1)^{n+1} \\
& \left|\frac{\partial^{m}}{\partial s^{m}}\left(\mathbf{T}_{a} J\right)(s, t)\right| \leqslant\left(\frac{\lambda}{6}\right)^{1+n} \frac{t^{2 n+1}}{(2 n+1) !}(2 L+1)^{n+1}
\end{aligned}
$$


These together with (3.36) imply that

$$
\left|\frac{\partial^{m}}{\partial s^{m}} G^{n+1}(s, t)\right| \leqslant \frac{\lambda^{1+n}}{3} \frac{t^{2 n+1}}{(2 n+1) !}(2 L+1)^{n+1},
$$

hence

$$
\sum_{n=1}^{+\infty} \frac{\partial^{m}}{\partial s^{m}} G^{n}(s, t) \text { is uniformly convergent in } \mathcal{T}_{0} .
$$

The same approach shows that the series

$$
\sum_{n=1}^{+\infty} \frac{\partial^{m+1}}{\partial t \partial s^{m}} G^{n}(s, t) \text { is uniformly convergent in } \mathcal{T}_{0} .
$$

We define

$$
G(s, t):=\sum_{n=1}^{+\infty} G^{n}(s, t),
$$

which is the solution of (3.12) (see [9, page 1691]). First, we estimate $|G|$ from (3.52), (3.53), and (3.55):

$$
|G(s, t)| \leqslant \frac{\sqrt{(2 L+1) \lambda}}{3} e^{\sqrt{(2 L+1) \lambda} t}, \text { in } \mathcal{T}_{0}
$$

Hence

$$
|G(s, t)| \leqslant \frac{e^{(1+L) \sqrt{(2 L+1) \lambda}}}{3} \leqslant e^{(1+L)^{2} \sqrt{\lambda}}, \text { in } \mathcal{T}_{0}
$$

It only remains to prove that $G(s, t) \in C^{3}\left(\mathcal{T}_{0}\right)$. Actually, from (3.53) and (3.54) we know that it suffices to prove $G_{t t}, G_{t t s}, G_{t t t} \in C^{0}\left(\mathcal{T}_{0}\right)$. We know from (2.15) that $G_{t t s} \in C^{0}\left(\mathcal{T}_{0}\right)$. As for $G_{t t}$ and $G_{t t t}$, thanks to (3.12), we get

$$
\begin{aligned}
6 G_{t}(s, t)= & \lambda(2 L-s-2 t)-\int_{0}^{t} \int_{0}^{\tau}\left(2 G_{s s s}+2 G_{s}+\lambda G\right)(2 L-t, \xi) d \xi d \tau \\
& +\int_{s}^{2 L-t} \int_{0}^{t}\left(2 G_{s s s}+2 G_{s}+\lambda G\right)(\eta, \xi) d \xi d \eta \\
6 G_{t t}(s, t)= & \lambda+\int_{0}^{t} \int_{0}^{\tau}\left(2 G_{s s s s}+2 G_{s s}+\lambda G_{s}\right)(2 L-t, \xi) d \xi d \tau \\
& -\int_{0}^{t}\left(2 G_{s s s}+2 G_{s}+\lambda G\right)(2 L-t, \xi) d \xi-\int_{0}^{t}\left(2 G_{s s s}+2 G_{s}+\lambda G\right)(2 L-t, \xi) d \xi \\
& +\int_{s}^{2 L-t}\left(2 G_{s s s}+2 G_{s}+\lambda G\right)(\eta, t) d \eta, \\
6 G_{t t t}(s, t)= & -\int_{0}^{t} \int_{0}^{\tau}\left(2 G_{s s s s}+2 G_{s s s}+\lambda G_{s s}\right)(2 L-t, \xi) d \xi d \tau+\int_{0}^{t}\left(2 G_{s s s s}+2 G_{s s}+\lambda G_{s}\right)(2 L-t, \xi) d \xi \\
& -2\left(2 G_{s s s}+2 G_{s}+\lambda G\right)(2 L-t, t) d \xi+2 \int_{0}^{t}\left(2 G_{s s s s}+2 G_{s s}+\lambda G_{s}\right)(2 L-t, \xi) d \xi \\
& +\int_{s}^{2 L-t}\left(2 G_{s s s t}+2 G_{s t}+\lambda G_{t}\right)(\eta, t) d \eta-\left(2 G_{s s s}+2 G_{s}+\lambda G\right)(2 L-t, t) .
\end{aligned}
$$

The above formulas together with (3.53)-(3.54) give the continuity of $G_{t t}$ and of $G_{t t t}$. Hence we complete the proof of Lemma 3. 
Remark 6. As we can see from [23, 39] for the heat equation, the $L^{\infty}{ }_{\text {-norm }}$ of the kernel $k_{\lambda}$ is of the form $e^{C \sqrt{\lambda}}$. One may naturally ask if the sharp estimate on the $L^{\infty}$-norm of kernel $k_{\lambda}$ is of the form $e^{C \sqrt[3]{\lambda}}$ for the KdV case, as KdV is of order 3. However, we do not know how to get such estimates.

At last, it remains to give the proof of Lemma 2.

Proof of Lemma 2. It is equivalent to prove the following statement:

Given $u_{0} \in L^{2}(0, L), \lambda>0, s>0$, one has

$$
\begin{gathered}
\kappa(t):=\int_{0}^{L} k_{\lambda}(0, \cdot)\left(\Pi_{\lambda}^{-1} S_{\lambda}(t) \Pi_{\lambda} u_{0}\right)(\cdot) d y \in L^{2}(0, s), \text { and } \\
u(t):=\Pi_{\lambda}^{-1} S_{\lambda}(t) \Pi_{\lambda} u_{0} \text { is the solution of }(2.1) \text { on }[0, s] \text { with } \tilde{h}=0 .
\end{gathered}
$$

We only need to prove the case where $u_{0} \in \Pi_{\lambda}^{-1} \mathcal{D}\left(\mathcal{A}_{\lambda}\right)$, since standard approximation methods then lead to the general case of $(S)$. From Section 2.3, we know that

$$
\omega(t):=S_{\lambda}(t) \Pi_{\lambda} u_{0} \in C^{0}\left([0, s] ; \mathcal{D}\left(\mathcal{A}_{\lambda}\right)\right) \cap C^{1}\left([0, s] ; L^{2}(0, L)\right),
$$

which shows that $\kappa(t) \in C^{0}([0, s])$. Direct calculations, based on (2.10), show that (similar to page 1690 in [9])

$$
\begin{aligned}
u_{t}= & \omega_{t}+\int_{x}^{L}\left(l_{y}+l_{y y y}-\lambda l\right)(x, z) \omega(z) d z \\
& -l_{y}(x, x) \omega_{x}(x)+l_{y y}(x, x) \omega(x) \\
u_{x}= & \omega_{x}(x)+\int_{x}^{L} l_{x}(x, z) \omega(z) d z \\
u_{x x}= & \omega_{x x}(x)-l_{x}(x, x) \omega(x)+\int_{x}^{L} l_{x x}(x, z) \omega(z) d z \\
u_{x x x}= & \omega_{x x x}-\left(l_{x x}(x, x)+l_{x y}(x, x)\right) \omega(x) \\
& -l_{x}(x, x) \omega_{x}(x)-l_{x x}(x, x) \omega(x)+\int_{x}^{L} l_{x x x}(x, z) \omega(x) d z,
\end{aligned}
$$

all these calculations hold on $C^{0}\left([0, T] ; L^{2}(0, L)\right)$. From (3.59)-(3.62) and (2.10), we know that

$$
\begin{gathered}
u(t, x) \in C^{1}\left([0, s] ; L^{2}(0, L)\right) \cap C^{0}\left([0, s] ; H^{3}(0, L)\right), \\
u_{t}+u_{x}+u_{x x x}=0, \text { in } L^{2}(0, L), \\
u(t, L)=u_{x}(t, L)=0, \\
u(t, 0)=\kappa(t),
\end{gathered}
$$

which show that $u$ satisfies Definition 1 .

Remark 7. In fact, by using (2.9) and the hidden inequality (2.35), we can also prove that $\kappa(t) \in H^{1}(0, s)$ with its norm controlled by $\left\|u_{0}\right\|_{L^{2}(0, L)}$. 


\section{Null controllability}

Finally, we are able to prove the null controllability (Theorem 1) by constructing a piecewise continuous bounded control. The construction is explained in Section 3. Thanks to Lemma 2 and (3.1)-(3.8), we only need to find good sequences $\left\{\lambda_{n}\right\}_{n \in \mathbb{N}}$ and $\left\{t_{n}\right\}_{n \in \mathbb{N}}$ such that:

$$
\begin{gathered}
\lim _{n \rightarrow+\infty} e^{-s_{n}} \prod_{k=0}^{n}\left(\left\|\Pi_{\lambda_{k}}^{-1}\right\|\left\|\Pi_{\lambda_{k}}\right\|\right)=0 \\
\lim _{n \rightarrow+\infty} e^{-s_{n}}\left\|k_{\lambda_{n}}(0, \cdot)\right\|_{L^{2}(0, L)} \prod_{k=0}^{n}\left(\left\|\Pi_{\lambda_{k}}^{-1}\right\|\left\|\Pi_{\lambda_{k}}\right\|\right)=0
\end{gathered}
$$

and that

$$
\left.u(t)\right|_{0 \leqslant t \leqslant T} \text { is a solution of (2.1) with } \tilde{h}=0, \kappa(t) \text { given by }(3.5) \text {. }
$$

Thanks to Lemma 2, from Definition 1, (3.7), (3.8), (4.1), and (4.2), one can easily deduce that $\left.u(t)\right|_{0 \leqslant t \leqslant T}$ is the solution of (1.1). It remains to prove that (4.1)-(4.2) hold.

From the definition of $\Pi_{\lambda}$ and $\Pi_{\lambda}^{-1},(2.19)-(2.20)$, we know that

$$
\begin{gathered}
\left\|\Pi_{\lambda}\right\|_{L^{2}(0, L) \rightarrow L^{2}(0, L)} \leqslant\left(1+L\left\|k_{\lambda}\right\|_{C^{0}(\mathcal{T})}\right) \leqslant e^{2(1+L)^{2} \sqrt{\lambda}} \\
\left\|\Pi_{\lambda}^{-1}\right\|_{L^{2}(0, L) \rightarrow L^{2}(0, L)} \leqslant\left(1+L\left\|l_{\lambda}\right\|_{C^{0}(\mathcal{T})}\right) \leqslant e^{2(1+L)^{2} \sqrt{\lambda}} \\
\left\|k_{\lambda}(0, \cdot)\right\|_{L^{2}(0, L)} \leqslant \sqrt{L}\left\|l_{\lambda}\right\|_{C^{0}(\mathcal{T})} \leqslant e^{2(1+L)^{2} \sqrt{\lambda}}
\end{gathered}
$$

where Lemma 3 is used. Hence it suffices to select $\left\{\lambda_{n}\right\}_{n \in \mathbb{N}}$ and $\left\{t_{n}\right\}_{n \in \mathbb{N}}$ such that

$$
e^{-s_{n}} \prod_{k=0}^{n} e^{6(1+L)^{2} \sqrt{\lambda_{k}}} \longrightarrow 0 .
$$

Inspired by the choices given by Coron and Nguyen in [23, Proposition 1], we select $t_{n}:=$ $T-1 / n^{2}$ and $\lambda_{n}:=2 n^{8}$. One easily verifies that (4.7) holds, which completes the proof.

Remark 8. To deal with the heat equations (by using backstepping approach), one needs to study the wave equation instead, which is already well investigated. In this article, we study the KdV system which has an order of 3. Hence the kernel system (see (A.1)) becomes a third order "wave-like" equation. For this reason, we encountered some difficulties: Lemma 3 for estimation and Proposition 1 for uniqueness. We believe that the Coron-Nguyen method, as well as the techniques introduced in this paper, could be used for other systems whose order is greater than 3. As we know, the backstepping method is well used on the rapid stablization problem of first-order hyperbolic systems (see [38]). Unfortunately, as they are of order one, we are not sure if some good estimates could be obtained for the null controllability or even the finite time stabilization. However, looking for [25, 37, 42], this might be possible for quasilinear hyperbolic systems.

\section{$5 \quad$ Further comments}

The above procedure has the following advantages.

- The null controllability is precisely obtained by an explicit piecewise continuous (actually piecewise $H^{1}$ ) bounded control instead of some unknown $H^{1 / 3}$ control. 
- The well-posedness results and the estimates investigated in this paper should allow smalltime stabilization to be proven (instead of rapid stabilization in [9]).

- The backstepping approach as used in [23] together with the techniques introduced in this paper could be applied to more models, as the backstepping method was widely used in the last 20 years for different models.

We also want to point out a list of open problems which could be further studied.

- The (global or local) controllability and the (global or local) small-time stabilization of the nonlinear $\mathrm{KdV}$ equations.

Based on the linear result and the Kato smoothing effect, we may expect the local controllability by standard perturbation. Actually, as it is shown in $[5,9,21,50]$ that the backstepping method can be directly used to treat the nonlinear case. But as the main purpose of this paper is to extend the new method found by Coron and Nguyen to more general models (as we stated in the Introduction), we do not consider nonlinear cases here.

However, since there is only one scalar control on the boundary, the global controllability in small time is a real challenge and might be false, as it is the case for the Burgers equations [34]. If there are more controls there are several global controllability results in small time for the nonlinear $\mathrm{KdV}$ equations [11] and for the Burgers equations [12, 26, 28, 29, 32, 48, 49]. Note that, using the backstepping approach, [26] allows to recover the global controllability result of [12] obtained by means of the return method. It would be interesting to see if [26] can be adapted to nonlinear KdV equations.

- In [40, 41], using a "microlocal" approach and Carleman's inequalities, Lebeau and Robbiano proved some $e^{C \sqrt{\lambda}}$ type estimates and then deduced from these estimates the controllability of the heat equation. From a "global" point of view, we also obtain the $e^{C \sqrt{\lambda}}$ type estimates (Lemma 3), and also get the controllability.

An important and interesting question: is there any connection between the backstepping method, Lebeau-Robbiano's strategy, Carleman inequalities, and small-time (local) stabilization?

- Let us also recall open problems raised in Remark 5 about the analytic regularity of the kernel and Remark 6 about sharp kernel estimates. For the sharp $L^{\infty}$-norm (or even $C^{n_{-}}$ norm) estimates, considering [14], perhaps it would be more natural to consider Gevrey class regularity instead of the analytic regularity.

Acknowledgments. The author would like to thank Jean-Michel Coron for having attracted his attention to this problem, for his constant support, and for fruitful discussions. He also thanks Amaury Hayat, Qi Lü, Peipei Shang, Daniel Wong, Bingyu Zhang, and Christophe Zhang for discussions on this problem. This work was supported by LIASFMA and ANR Project Finite4SoS (ANR 15-CE23-0007).

\section{A Appendix: Proof of Proposition 1}

In this part, we give the proof of the uniqueness of the solution to equation (2.24). As the function is defined in the triangle $\mathcal{T}$, we extend $l_{0}$ by 0 in the lower triangle $[0, L] \times[0, L] \backslash \mathcal{T}$, and denote by $h$ the extended function. Since on the diagonal $x=y, C^{3}(\mathcal{T})$ function $l_{0}$ satisfies

$$
l_{0 x}=l_{0 y}=l_{0 x x}=l_{0 x y}=l_{0 y y}=0,
$$


the extended function is $H^{3}([0, L] \times[0, L])$. Moreover, $h$ satisfies

$$
\begin{cases}h_{x x x}+h_{y y y}+h_{x}+h_{y}=0 & \text { in }[0, L] \times[0, L], \\ h(x, L)=0 & \text { on }[0, L], \\ h(x, 0)=h_{y}(x, 0)=h_{y y}(x, 0)=0 & \text { on }[0, L], \\ h(L, y)=h_{x}(L, y)=h_{x x}(L, y)=0 & \text { on }[0, L] .\end{cases}
$$

By simple change of variables, $\tilde{x}=L-x$ and $\tilde{y}=L-y$, it suffices to prove that the solution $h \in H^{3}([0, L] \times[0, L])$ of

$$
\begin{cases}h_{x x x}+h_{y y y}+h_{x}+h_{y}=0 & \text { in }[0, L] \times[0, L], \\ h(x, 0)=0 & \text { on }[0, L], \\ h(x, L)=h_{y}(x, L)=h_{y y}(x, L)=0 & \text { on }[0, L] \\ h(0, y)=h_{x}(0, y)=h_{x x}(0, y)=0 & \text { on }[0, L]\end{cases}
$$

is 0 .

As (A.2) is similar to the wave equation, it is natural to consider eigenfunctions of the operator (with respect to $y$ variable),

$$
\begin{gathered}
\mathcal{A}_{y}: \mathcal{D}\left(\mathcal{A}_{y}\right) \subset L^{2}(0, L) \rightarrow L^{2}(0, L), \\
\mathcal{D}\left(\mathcal{A}_{y}\right):=\left\{f \in H^{3}(0, L) ; f(0)=f(L)=f_{y}(L)=0\right\}, \\
\mathcal{A}_{y} f:=-f_{y}-f_{y y y}, \forall f \in \mathcal{D}\left(\mathcal{A}_{y}\right) .
\end{gathered}
$$

If the eigenfunctions, $\left\{\varphi_{n}(y)\right\}_{n}$, form a Riesz basis of $L^{2}(0, L)$, then the Fourier series decomposition

$$
h(x, y)=\sum_{n} \phi_{n}(x) \cdot \varphi_{n}(y)
$$

easily infers the uniqueness required. Unfortunately, this operator is a non-self-adjoint operator and eigenfunctions do not form a Riesz basis, see [53].

Another idea is to investigate the completeness of eigenfunctions, $\left\{\psi(y)_{n}\right\}_{n}$, of the adjoint operator $\mathcal{A}_{y}^{*}$,

$$
\begin{aligned}
& \mathcal{A}_{y}^{*}: \mathcal{D}\left(\mathcal{A}_{y}^{*}\right) \subset L^{2}(0, L) \rightarrow L^{2}(0, L), \\
\mathcal{D}\left(\mathcal{A}_{y}^{*}\right):= & \left\{f \in H^{3}(0, L) ; f(0)=f(L)=f_{y}(0)=0\right\}, \\
& \mathcal{A}_{y}^{*} f:=-f_{y}-f_{y y y}, \forall f \in \mathcal{D}\left(\mathcal{A}_{y}^{*}\right) .
\end{aligned}
$$

Actually, suppose that $\left\{\psi_{n}(y)\right\}_{n}$ is an eigenfunction of the adjoint operator $\mathcal{A}_{y}^{*}$, then from (A.2) as well as the boundary conditions of $h$ and $\psi$ one can deduce that

$$
\left(\partial_{x x x}+\partial_{x}-\lambda_{n}\right)\left\langle\psi_{n}(\cdot), h(x, \cdot)\right\rangle_{L^{2}(0, L)}=0 .
$$

Combine (A.10) with the fact that

$$
\left\langle\psi_{n}(\cdot), h(0, \cdot)\right\rangle_{L^{2}(0, L)}=\partial_{x}\left\langle\psi_{n}(\cdot), h(0, \cdot)\right\rangle_{L^{2}(0, L)}=\partial_{x x}\left\langle\psi_{n}(\cdot), h(0, \cdot)\right\rangle_{L^{2}(0, L)}=0,
$$

we obtain

$$
\left\langle\psi_{n}(\cdot), h(x, \cdot)\right\rangle_{L^{2}(0, L)}=0, \quad \forall x \in[0, L]
$$


If $\left\{\psi_{n}(y)\right\}_{n}$ is complete in $L^{2}(0, L)$, then $h(x, 0)$ is 0 . However, we don't know the completeness of the eigenfunctions $\left\{\psi_{n}(y)\right\}_{n}$.

More generally, one could consider eigenfunctionals or even generalized eigenfunctions, following Gel'fand and the coauthors [30,31]. More precisely, in the non-self-adjoint cases it is not always possible to expand a function as the sum of eigenfunctions. In order to avoid this problem, one uses different generalisations of eigenfunctions.

For example, the generalisation introduced by Fokas, augmented eigenfunctions, which is itself a generalisation of Gel'fand's eigenfunctions (allow the appearance of remainder functionals). This generalisation turns out to be a powerful tool to investigate the initial-boundary value problem (IBVP). One can find an almost complete investigation from the papers [46, 47, 59]. In general, let $\Phi$ be a function space defined on the closure of a real interval $I$ with sufficient smoothness and decay conditions, $\mathcal{L}$ be a linear operator defined on $\Phi$. Let $\gamma$ be an oriented contour in $\mathbb{C}$, and let $\mathbf{E}=\left\{\mathbf{E}_{\lambda}: \lambda \in \gamma\right\}$ be a family of functionals (imagine as a family of eigenfunction when $\gamma$ is only defined on a discrete set). Then the corresponding remainder functionals $\mathbf{R}_{\lambda} \in \Phi^{\prime}$ with respect to eigenvalues $\lambda$ is

$$
\mathbf{R}_{\lambda}(\phi):=\lambda^{n} \mathbf{E}_{\lambda}(\phi)-\mathbf{E}_{\lambda}(\mathcal{L} \phi), \quad \forall \phi \in \Phi, \forall \lambda \in \gamma .
$$

One is interested in the cases in which one of the following two conditions is satisfied,

$$
\int_{\lambda} e^{i \lambda x} \mathbf{R}_{\lambda}(\phi) d \lambda=0, \quad \forall \phi \in \Phi, \forall x \in I
$$

or

$$
\int_{\lambda} \frac{e^{i \lambda x}}{\lambda^{n}} \mathbf{R}_{\lambda}(\phi) d \lambda=0, \quad \forall \phi \in \Phi, \forall x \in I,
$$

where (A.14) (resp. (A.15)) is called the type I (resp. type II) condition of augmented eigenfunctions of $\mathcal{L}$ up to the integration along $\gamma$.

As we can see above, the study of augmented eigenfunctions involves complicated asymptotic calculations. In Fokas' work this method is only used to study the evolution equations based on a good transform pair, which does not seem to be a good (easy) option to our problem (A.2). Instead of augmented eigenfunctions, Locker [47] also considered the generalized eigenspace $\mathcal{E}_{\mathcal{G}}$ given by

$$
\bigcup \mathcal{N}\left(\left(\lambda_{i} I-\mathcal{L}\right)^{m_{i}}\right) \text {, union for all } m_{i} \in \mathbb{N} \text {, and } \lambda_{i} \text { eigenvalues, }
$$

where $\mathcal{L}$ denotes the operator, $\mathcal{N}$ denotes the kernel. More precisely, to the linearized $\mathrm{KdV}$ operator he proved the following.

Theorem 2. Let $L>0$. For the differential operator $\mathcal{L} f:=f_{x x x}+a f_{x}$ with boundary conditions

$$
\begin{gathered}
f(0)=f(L)=0, \\
f_{x}(0)+\beta f_{x}(L)=0,
\end{gathered}
$$

the generalized eigenfunction space $\mathcal{E}_{\mathcal{G}}$ is complete in $L^{2}(0, L)$ space when $\beta \neq 0$.

Remark 9. When $\beta=0$, it does not seem to be known whether generalized eigenfunction space $\mathcal{E}_{\mathcal{G}}$ is complete in $L^{2}(0, L)$. This is one of the reasons that much more complicated augmented eigenfunctions are introduced (the other reasons are about the regularities and some more general boundary conditions). Actually, this case can be regarded as a limit of the cases when the coupling constant $\beta$ approaches 0 .

In fact, in [47] Locker only considered the operator $\mathcal{L} f:=f_{x x x}$. One can easily verify with the same proof that the same result holds when there is an additional $f_{x}$ in the operator. 
In order to solve our problem, we use another kind of generalized eigenfunctions, which is more general than $\mathcal{E}_{\mathcal{G}}$ but is less general than augmented eigenfunctions, namely eigenfunctions and associated functions (e.a.f.). The definition of e.a.f., which is defined on equations with $\lambda$ as parameter, is rather complicated. One can see [51, chapter 1] and [52] for precise description on this subject.

With eigenfunctions and associated functions, Shkalikov in [58] proved the following theorem.

Theorem 3. The eigenfunctions and associated functions of the boundary-value problem generated by an ordinary differential equation with separated boundary conditions

$$
\begin{gathered}
l(y)-\lambda^{n} y=y^{(n)}+p_{n-2}(x) y^{(n-2)}+\ldots+p_{0}(x) y-\lambda^{n} y=0, \\
U_{j}(y)=\sum_{k=0}^{n-1} \alpha_{j k} y^{(k)}(0)=0, \text { with } j=1,2, \ldots, l, \\
U_{j}(y)=\sum_{k=0}^{n-1} \beta_{j k} y^{(k)}(L)=0, \text { with } j=1,2, \ldots, n-l,
\end{gathered}
$$

form a complete system in the space $L^{2}[0, L]$, where $p_{i}(x)$ are arbitrary summable functions, and $l>n-l>0$.

Applying Theorem 3 to our case (linearised KdV), we get

Corollary 1. For the ordinary differential equation with separated boundary conditions

$$
\begin{aligned}
\mu(f)=l(f)-\lambda^{3} f & =f_{y y y}+f_{y}-\lambda^{3} f=0, \\
U_{1}(f) & =f(0)=0, \\
U_{2}(f) & =f_{y}(0)=0, \\
U_{3}(f) & =f(L)=0,
\end{aligned}
$$

the eigenfunctions and associated functions form a complete system in the space $L^{2}[0, L]$.

Finally, we are able to prove Proposition 1.

Proof of Proposition 1. Let us consider the boundary-value problem (A.21)-(A.24). Let $\lambda_{0}$ be an eigenvalue, and let $\varphi_{0}(y)=\varphi(y)$ an eigenfunction for the eigenvalue $\lambda_{0}$. The associated functions associated with the eigenfunction $\varphi(y)$ are given by the functions

$$
\varphi_{1}(y), \varphi_{2}(y), \ldots, \varphi_{k}(y)
$$

These functions satisfy (the boundary conditions)

$$
U_{1}\left(\varphi_{i}\right)=U_{2}\left(\varphi_{i}\right)=U_{3}\left(\varphi_{i}\right)=0, \quad \forall i=0,1, \ldots, k,
$$

and, for $\lambda=\lambda_{0}$, the following relations

$$
\mu\left(\varphi_{i}\right)+\frac{1}{1 !} \frac{\partial}{\partial \lambda} \mu\left(\varphi_{i-1}\right)+\ldots+\frac{1}{i !} \frac{\partial^{i}}{\partial \lambda^{i}} \mu\left(\varphi_{0}\right)=0, \quad \forall i=0,1, \ldots, k .
$$

Now we prove that for all those functions (e.a.f.), we have

$$
\left\langle h(x, \cdot), \varphi_{i}(\cdot)\right\rangle_{L^{2}(0, L)}=0, \quad \forall x \in[0, L], \quad \forall i=0,1, \ldots, k .
$$


At first, for $\varphi_{0}$, as what we have done in (A.12), clearly

$$
\left\langle h(x, \cdot), \varphi_{0}(\cdot)\right\rangle_{L^{2}(0, L)}=0, \quad \forall x \in[0, L] .
$$

For $\varphi_{1}$, equation (A.26) shows that

$$
\left(\varphi_{1}\right)_{y y y}+\left(\varphi_{1}\right)_{y}-\lambda_{0}^{3} \varphi_{1}-3 \lambda_{0}^{2} \varphi_{0}=0 .
$$

Hence from (A.2), (A.25), (A.28), and (A.29) we get

$$
\begin{aligned}
0 & =\left\langle\left(\partial_{x}^{3}+\partial_{x}+\partial_{y}^{3}+\partial_{y}\right) h(x, \cdot), \varphi_{1}(\cdot)\right\rangle_{L^{2}(0, L)} \\
& =\left(\partial_{x}^{3}+\partial_{x}\right)\left\langle h(x, \cdot), \varphi_{1}(\cdot)\right\rangle_{L^{2}(0, L)}-\left\langle h(x, \cdot),\left(\partial_{y}^{3}+\partial_{y}\right) \varphi_{1}(\cdot)\right\rangle_{L^{2}(0, L)} \\
& =\left(\partial_{x}^{3}+\partial_{x}\right)\left\langle h(x, \cdot), \varphi_{1}(\cdot)\right\rangle_{L^{2}(0, L)}-\left\langle h(x, \cdot),-\lambda_{0}^{3} \varphi_{1}(\cdot)-3 \lambda_{0}^{2} \varphi_{0}(\cdot)\right\rangle_{L^{2}(0, L)} \\
& =\left(\partial_{x}^{3}+\partial_{x}+\lambda_{0}^{3}\right)\left\langle h(x, \cdot), \varphi_{1}(\cdot)\right\rangle_{L^{2}(0, L)}
\end{aligned}
$$

By using the the fact that

$$
\left\langle h(0, \cdot), \varphi_{1}(\cdot)\right\rangle_{L^{2}(0, L)}=\partial_{x}\left\langle h(0, \cdot), \varphi_{1}(\cdot)\right\rangle_{L^{2}(0, L)}=\partial_{x x}\left\langle h(0, \cdot), \varphi_{1}(\cdot)\right\rangle_{L^{2}(0, L)}=0,
$$

we get

$$
\left\langle h(x, \cdot), \varphi_{1}(\cdot)\right\rangle_{L^{2}(0, L)}=0, \quad \forall x \in[0, L] .
$$

Repeating this procedure we get (A.27), which combined with Corollary 1 shows that

$$
h(x, \cdot)=0, \quad \forall x \in[0, L] .
$$

Hence the proof of Proposition 1 is completed.

Remark 10. For the y-variable, we only used 3 boundary conditions in the proof to deduce the uniqueness of the solution $h: h(x, L)=h_{y}(x, L)=h(x, 0)=0$. This is natural, since once we consider 4 boundary conditions (for a third order differential operator), the eigenfunctions could never become a basis.

We may also wonder, if we can get the uniqueness of $h$ by using the other 3 boundary conditions in y-variable: $h(x, L)=h_{y}(x, L)=h_{y y}(x, L)=0$ ? Unfortunately, Theorem 3 can not be applied for these 3 boundary conditions: we observe from (A.19) and (A.20) that there should be boundary conditions on both side. Hence, it is difficult to get the uniqueness of $h$ by using the Carleman estimate, see [1, Chapter 4], [6] and [35], though the Carleman estimate is a standard way to solve the unique continuation problem.

\section{References}

[1] Fatiha Alabau-Boussouira, Roger Brockett, Olivier Glass, Jérôme Le Rousseau, and Enrique Zuazua. Control of partial differential equations, volume 2048 of Lecture Notes in Mathematics. Springer, Heidelberg; Fondazione C.I.M.E., Florence, 2012. Lectures from the CIME Course held in Cetraro, July 19-23, 2010, Edited by Piermarco Cannarsa and Jean-Michel Coron, Fondazione CIME/CIME Foundation Subseries.

[2] Georges Bastin and Jean-Michel Coron. Stability and boundary stabilization of 1-D hyperbolic systems, volume 88 of Progress in Nonlinear Differential Equations and their Applications. Birkhäuser/Springer, [Cham], 2016. Subseries in Control. 
[3] Jerry L. Bona, Shu Ming Sun, and Bing-Yu Zhang. A nonhomogeneous boundary-value problem for the Korteweg-de Vries equation posed on a finite domain. Comm. Partial Differential Equations, 28(7-8):1391-1436, 2003.

[4] Dejan M. Bošković, Andras Balogh, and Miroslav Krstić. Backstepping in infinite dimension for a class of parabolic distributed parameter systems. Math. Control Signals Systems, 16(1):44-75, 2003.

[5] Roberto A. Capistrano-Filho and Fernando A. Gallego. Asymptotic behavior of Boussinesq system of KdV-KdV type. J. Differential Equations, 265(6):2341-2374, 2018.

[6] Torsten Carleman. Sur un problème d'unicité pur les systèmes d'équations aux dérivées partielles à deux variables indépendantes. Ark. Mat., Astr. Fys., 26(17):9, 1939.

[7] Eduardo Cerpa. Exact controllability of a nonlinear Korteweg-de Vries equation on a critical spatial domain. SIAM J. Control Optim., 46(3):877-899 (electronic), 2007.

[8] Eduardo Cerpa. Control of a Korteweg-de Vries equation: a tutorial. Math. Control Relat. Fields, 4(1):45-99, 2014.

[9] Eduardo Cerpa and Jean-Michel Coron. Rapid stabilization for a Korteweg-de Vries equation from the left Dirichlet boundary condition. IEEE Trans. Automat. Control, 58(7):1688-1695, 2013.

[10] Eduardo Cerpa and Emmanuelle Crépeau. Boundary controllability for the nonlinear Korteweg-de Vries equation on any critical domain. Ann. Inst. H. Poincaré Anal. Non Linéaire, 26(2):457-475, 2009.

[11] Marianne Chapouly. Global controllability of a nonlinear Korteweg-de Vries equation. Commun. Contemp. Math., 11(3):495-521, 2009.

[12] Marianne Chapouly. Global controllability of nonviscous and viscous Burgers-type equations. SIAM J. Control Optim., 48(3):1567-1599, 2009.

[13] Jixun Chu, Jean-Michel Coron, and Peipei Shang. Asymptotic stability of a nonlinear Korteweg-de Vries equation with critical lengths. J. Differential Equations, 259(8):40454085, 2015.

[14] Jixun Chu, Jean-Michel Coron, Peipei Shang, and Shu-Xia Tang. Gevrey Class Regularity of a Semigroup Associated with a Nonlinear Korteweg-de Vries Equation. Chin. Ann. Math. Ser. B, 39(2):201-212, 2018.

[15] Jean-Michel Coron. Control and nonlinearity, volume 136 of Mathematical Surveys and Monographs. American Mathematical Society, Providence, RI, 2007.

[16] Jean-Michel Coron. Stabilization of control systems and nonlinearities. In Proceedings of the 8th International Congress on Industrial and Applied Mathematics, pages 17-40. Higher Ed. Press, Beijing, 2015.

[17] Jean-Michel Coron and Emmanuelle Crépeau. Exact boundary controllability of a nonlinear KdV equation with critical lengths. J. Eur. Math. Soc. (JEMS), 6(3):367-398, 2004.

[18] Jean-Michel Coron and Brigitte d'Andréa Novel. Stabilization of a rotating body beam without damping. IEEE Trans. Automat. Control, 43(5):608-618, 1998. 
[19] Jean-Michel Coron, Ludovick Gagnon, and Morgan Morancey. Rapid stabilization of 1-D linear Schrödinger equations. To appear in J. Math. Pures Appl. (9).

[20] Jean-Michel Coron, Long $\mathrm{Hu}$, and Guillaume Olive. Finite-time boundary stabilization of general linear hyperbolic balance laws via Fredholm backstepping transformation. Automatica, 84:95-100, 2017.

[21] Jean-Michel Coron and Qi Lü. Local rapid stabilization for a Korteweg-de Vries equation with a Neumann boundary control on the right. J. Math. Pures Appl. (9), 102(6):1080$1120,2014$.

[22] Jean-Michel Coron and Qi Lü. Fredholm transform and local rapid stabilization for a Kuramoto-Sivashinsky equation. J. Differential Equations, 259(8):3683-3729, 2015.

[23] Jean-Michel Coron and Hoai-Minh Nguyen. Null controllability and finite time stabilization for the heat equations with variable coefficients in space in one dimension via backstepping approach. Arch. Ration. Mech. Anal., 225(3):993-1023, 2017.

[24] Jean-Michel Coron, Ivonne Rivas, and Shengquan Xiang. Local exponential stabilization for a class of Korteweg-de Vries equations by means of time-varying feedback laws. Anal. PDE, 10(5):1089-1122, 2017.

[25] Jean-Michel Coron, Rafael Vazquez, Miroslav Krstic, and Georges Bastin. Local exponential $H^{2}$ stabilization of a $2 \times 2$ quasilinear hyperbolic system using backstepping. SIAM J. Control Optim., 51(3):2005-2035, 2013.

[26] Jean-Michel Coron and Shengquan Xiang. Small-time global stabilization of the viscous Burgers equation with three scalar controls. Preprint, 2018.

[27] Florent Di Meglio, Rafael Vazquez, and Miroslav Krstic. Stabilization of a system of $n+1$ coupled first-order hyperbolic linear PDEs with a single boundary input. IEEE Trans. Autom. Control, 58(12):3097-3111, 2013.

[28] Enrique Fernández-Cara and Sergio Guerrero. Null controllability of the Burgers system with distributed controls. Systems Control Lett., 56(5):366-372, 2007.

[29] Ludovick Gagnon. Lagrangian controllability of the 1-dimensional Korteweg-de Vries equation. SIAM J. Control Optim., 54(6):3152-3173, 2016.

[30] Israel M. Gel'fand and Georgi E. Shilov. Generalized functions. Vol. 3. AMS Chelsea Publishing, Providence, RI, 2016. Theory of differential equations, Translated from the 1958 Russian original [ MR0106410] by Meinhard E. Mayer, Reprint of the 1967 English translation [ MR0217416].

[31] Israel M. Gel'fand and Naum Ya. Vilenkin. Generalized functions. Vol. 4. AMS Chelsea Publishing, Providence, RI, 2016. Applications of harmonic analysis, Translated from the 1961 Russian original [ MR0146653] by Amiel Feinstein, Reprint of the 1964 English translation [ MR0173945].

[32] Olivier Glass and Sergio Guerrero. On the uniform controllability of the Burgers equation. SIAM J. Control Optim., 46(4):1211-1238, 2007. 
[33] Olivier Glass and Sergio Guerrero. Some exact controllability results for the linear KdV equation and uniform controllability in the zero-dispersion limit. Asymptot. Anal., 60(12):61-100, 2008.

[34] Sergio Guerrero and Oleg Yu. Imanuvilov. Remarks on global controllability for the Burgers equation with two control forces. Ann. Inst. H. Poincaré Anal. Non Linéaire, 24(6):897906, 2007.

[35] Lars Hörmander. On the uniqueness of the Cauchy problem under partial analyticity assumptions. In Geometrical optics and related topics (Cortona, 1996), volume 32 of Progr. Nonlinear Differential Equations Appl., pages 179-219. Birkhäuser Boston, Boston, MA, 1997.

[36] Long Hu, Florent Di Meglio, Rafael Vazquez, and Miroslav Krstic. Control of homodirectional and general heterodirectional linear coupled hyperbolic PDEs. IEEE Trans. Autom. Control, 61(11):3301-3314, 2016.

[37] Long Hu, Rafael Vasquez, Florent Di Meglio, and Miroslav Krstic. Boundary exponential stabilization of 1-d inhomogeneous quasilinear hyperbolic systems. Preprint, 2015.

[38] Miroslav Krstic and Andrey Smyshlyaev. Backstepping boundary control for first-order hyperbolic PDEs and application to systems with actuator and sensor delays. Systems Control Lett., 57(9):750-758, 2008.

[39] Miroslav Krstic and Andrey Smyshlyaev. Boundary control of PDEs, volume 16 of Advances in Design and Control. Society for Industrial and Applied Mathematics (SIAM), Philadelphia, PA, 2008. A course on backstepping designs.

[40] Gilles Lebeau and Luc Robbiano. Contrôle exact de l'équation de la chaleur. Comm. Partial Differential Equations, 20(1-2):335-356, 1995.

[41] Gilles Lebeau and Luc Robbiano. Contrôle exacte de l'équation de la chaleur. In Séminaire sur les Équations aux Dérivées Partielles, 1994-1995, pages Exp. No. VII, 13. École Polytech., Palaiseau, 1995.

[42] Ta Tsien Li. Global classical solutions for quasilinear hyperbolic systems, volume 32 of RAM: Research in Applied Mathematics. Masson, Paris; John Wiley \& Sons, Ltd., Chichester, 1994.

[43] Jacques-Louis Lions and Enrico Magenes. Non-homogeneous boundary value problems and applications. Vol. III. pages xii+308, 1973. Translated from the French by P. Kenneth, Die Grundlehren der mathematischen Wissenschaften, Band 183.

[44] Wei-Jiu Liu and Miroslav Krstic. Stability enhancement by boundary control in the Kuramoto-Sivashinsky equation. Nonlinear Anal. Ser. A: Theory Methods, 43(4):485-507, 2001.

[45] Weijiu Liu. Boundary feedback stabilization of an unstable heat equation. SIAM J. Control Optim., 42(3):1033-1043, 2003.

[46] John Locker. Spectral theory of non-self-adjoint two-point differential operators, volume 73 of Mathematical Surveys and Monographs. American Mathematical Society, Providence, RI, 2000. 
[47] John Locker. Eigenvalues and completeness for regular and simply irregular two-point differential operators. Mem. Amer. Math. Soc., 195(911):viii+177, 2008.

[48] Frédéric Marbach. An obstruction to small time local null controllability for a viscous Burgers' equation. To appear in Ann. Sci. Éc. Norm. Supér. (4).

[49] Frédéric Marbach. Small time global null controllability for a viscous Burgers' equation despite the presence of a boundary layer. J. Math. Pures Appl. (9), 102(2):364-384, 2014.

[50] Swann Marx and Eduardo Cerpa. Output feedback stabilization of the Korteweg-de Vries equation. Automatica J. IFAC, 87:210-217, 2018.

[51] Mark A. Naumark. Linear differential operators. Part I: Elementary theory of linear differential operators. Frederick Ungar Publishing Co., New York, 1967.

[52] Mark A. Nămark. Linear differential operators. Part II: Linear differential operators in Hilbert space. With additional material by the author, and a supplement by V. È. Ljance. Translated from the Russian by E. R. Dawson. English translation edited by W. N. Everitt. Frederick Ungar Publishing Co., New York, 1968.

[53] Vassilis G. Papanicolaou. An example where separation of variables fails. J. Math. Anal. Appl., 373(2):739-744, 2011.

[54] Gustavo Alberto Perla Menzala, C. F. Vasconcellos, and Enrique Zuazua. Stabilization of the Korteweg-de Vries Equation with localized damping. Q. Appl. Math., LX(1):111-129, 2002.

[55] Lionel Rosier. Exact boundary controllability for the Korteweg-de Vries equation on a bounded domain. ESAIM Control Optim. Calc. Var., 2:33-55 (electronic), 1997.

[56] Lionel Rosier. Control of the surface of a fluid by a wavemaker. ESAIM Control Optim. Calc. Var., 10(3):346-380 (electronic), 2004.

[57] Lionel Rosier and Bing-Yu Zhang. Control and stabilization of the Korteweg-de Vries equation: recent progresses. J. Syst. Sci. Complex., 22(4):647-682, 2009.

[58] Andrei A. Škalikov. The completeness of the eigen- and associated functions of an ordinary differential operator with nonregular splitting boundary conditions. Funkcional. Anal. $i$ Priložen., 10(4):69-80, 1976.

[59] David A. Smith and Athanassios S. Fokas. Evolution PDEs and augmented eigenfunctions. Finite interval. Preprint.

[60] Andrey Smyshlyaev, Eduardo Cerpa, and Miroslav Krstic. Boundary stabilization of a 1-D wave equation with in-domain antidamping. SIAM J. Control Optim., 48(6):4014-4031, 2010.

[61] Shuxia Tang, Jixun Chu, Peipei Shang, and Jean-Michel Coron. Local asymptotic stability of a KdV system with a two-dimensional center manifold. Adv. Nonlinear Anal., 2016. 